\title{
Lattice QCD and New Physics searches: present and future
}

\author{
L. Silvestrini ${ }^{* \dagger}$ \\ INFN, Sez. di Roma \\ P.le A. Moro, 5 \\ I-00185 Roma \\ Italy \\ E-mail: Luca.Silvestrini@romal.infn.it
}

We discuss the key role played by Lattice QCD in searching for New Physics in the flavour sector. We present the current status of the Unitarity Triangle analysis in the Standard Model and beyond, and outline future developments.

The XXVI International Symposium on Lattice Field Theory

July 14-19 2008

Williamsburg, Virginia, USA

\footnotetext{
* Speaker.

${ }^{\dagger}$ Member of the UTfit Collaboration.
} 


\section{Introduction}

Lattice QCD played a relevant role in the history the Unitarity Triangle (UT) fit since the very beginning $[1,2,3,4]$, allowing for predictions of the value of $\sin 2 \beta$ and $\Delta m_{s}$ before the advent of direct measurements. At the time when the $B$-factories had not started yet and inclusive measurements of $V_{u b}$ and $V_{c b}$ were rather rough, the "classical" UT analysis for the determination of $\bar{\rho}$ and $\bar{\eta}$ relied on the results of quenched lattice QCD simulations to relate the measured exclusive semileptonic $B$ decays, the $B_{d}-\bar{B}_{d}$ mixing amplitude, the lower bound on $B_{s}-\bar{B}_{s}$ oscillations and CP violation in $K^{0}-\bar{K}^{0}$ mixing to the CKM parameters. In spite of these caveat the prediction of $\sin 2 \beta$ in the years was quite stable, going from $\sin 2 \beta=0.65 \pm 0.12$ in 1995 [1] to $\sin 2 \beta=0.698 \pm 0.066$ in 2000 [4]. A similar situation is true for $\Delta m_{s}$, for which a first precise indirect determination from the other constraints of the UT fit was available since $1997\left([6.5,15.0] \mathrm{ps}^{-1}\right.$ at $68 \%$ probability and $\Delta m_{s}<22 \mathrm{ps}^{-1}$ at $95 \%$ probability) [3]. A compilation of the predictions for $\Delta m_{s}$ by various collaborations as a function of time is shown in Fig. 1. An upgraded version of our Standard Model (SM) "prediction" for $\Delta m_{s}$, obtained from an overall UT fit which makes use of all the latest input values and constraints, is given in Tab. $1: \Delta m_{s}^{\text {pred }}=(16.8 \pm 1.6) \mathrm{ps}^{-1}$. This is the number and uncertainty to compare with the CDF result $\Delta m_{s}^{\text {exp }}=(17.77 \pm 0.12) \mathrm{ps}^{-1}$ [9].

Lattice QCD also plays a crucial role in constraining NP contributions to flavour physics, in particular in $\Delta F=2$ processes, which give very strong bounds due to QCD enhancements of the additional operators arising beyond the SM $[10,11,12,13,14]$. To this aim, however, further efforts are strongly required, since only very few computations of matrix elements of non-SM operators are presently available $[15,16]$.

\section{SM UT fit}

A major advance in flavour physics in the past few years has been possible with the experimental determination of the UT angles, obtained by studying non-leptonic decays: the angle $\alpha$ from $B \rightarrow \pi \pi, B \rightarrow \pi \rho$ and $B \rightarrow \rho \rho$ decays [17]; the angle $\gamma$ from $B \rightarrow D^{(*)} K^{(*)}$ decays [18]; $2 \beta+\gamma$ from time-dependent asymmetries in $B \rightarrow D^{(*)} \pi(\rho)$ decays [19]; $\cos 2 \beta$ from $B_{d}^{0} \rightarrow J / \psi K_{S}^{* 0}$ [20]; $\beta$ from $B \rightarrow D^{0} \pi^{0}$ [21] and, finally, $\sin 2 \beta$ from the "golden mode" $B_{d}^{0} \rightarrow J / \psi K_{S}$ [22, 23]. These measurements allow a determination of $\bar{\rho}$ and $\bar{\eta}$ independently of the hadronic parameters computed on the lattice. The precision in constraining $\bar{\rho}$ and $\bar{\eta}$ from the angles is by now comparable to that obtained from lattice-related constraints. The latter include the information coming from semileptonic decays, namely $\left|V_{u b}\right| /\left|V_{c b}\right|, \varepsilon_{K}, \Delta m_{d}$ and $\Delta m_{s}$. In figure 2 we show the results of the global fit, while in figure 3 we compare the results of the fit from angles with the results of the lattice-related fit.

The consistency of the global fit can be read from Tab. 1, comparing the results obtained using all available information (Global Fit) with the "predictions" for several observables obtained without using the experimental information on a given observable (Fit Prediction). ${ }^{1}$ We see that:

- The indirect determination of the angles $\alpha$ and $\gamma$ is dominating the fit. Thus, the direct measurements play an important role only beyond the SM, as we shall see in the following.

\footnotetext{
${ }^{1}$ The input values used to obtain these results can be found at http://www . utfit.org.
} 


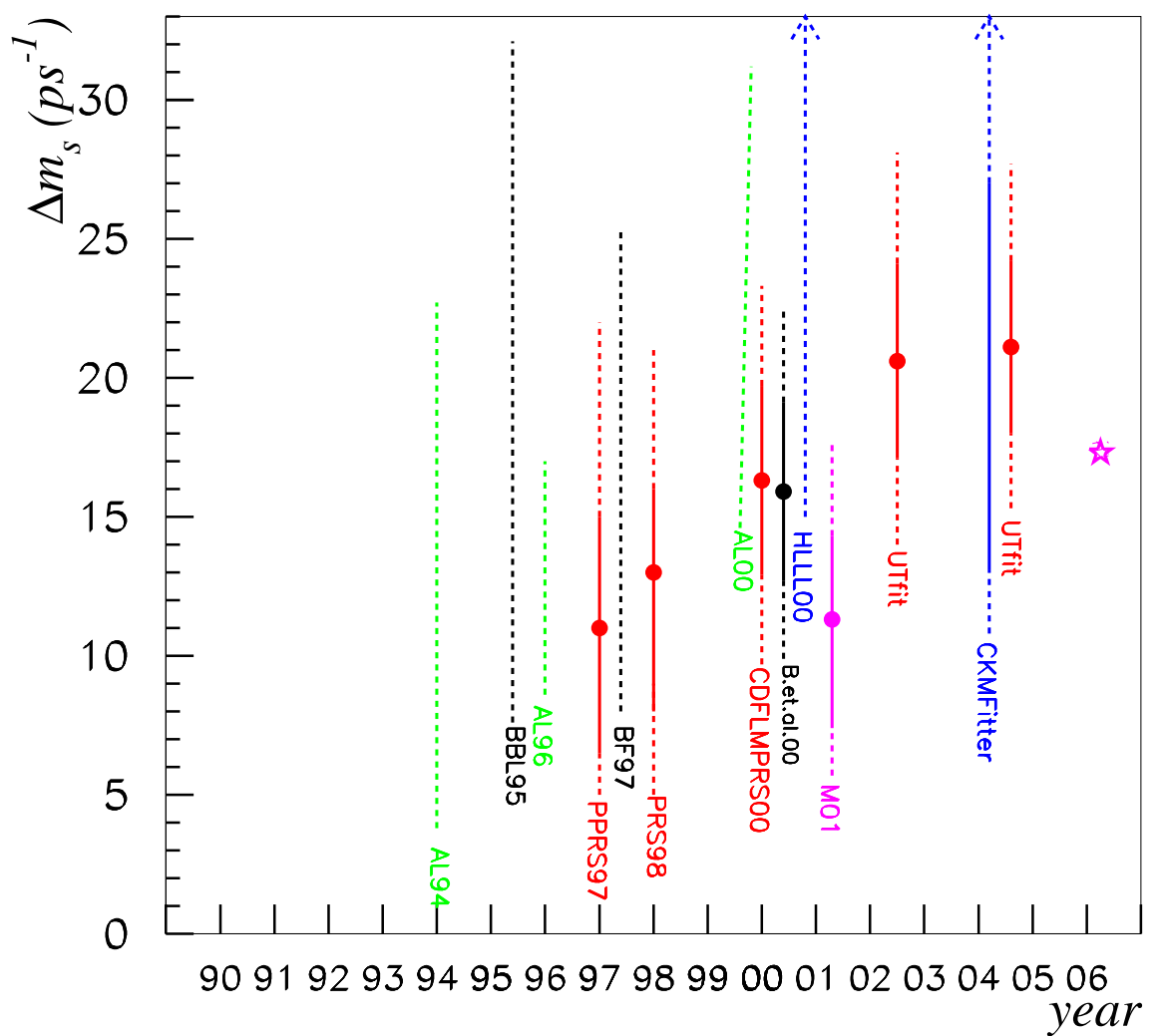

Figure 1: Evolution of the "indirect" determination of $\Delta m_{s}$ over the years. These determinations are given in $[3,4,5,6,7,8]$. From left to right, they correspond to the following papers: AL94 (Ali, London), BBL95 (Buchalla,Buras,Lautenbacher), AL96, PPRS97 (Paganini, Parodi, Roudeau, Stocchi), BF97 (Buras,Fleischer), PRS98 (Parodi,Roudeau,Stocchi), AL00, CDFLMPRS00 (Ciuchini et al.), B.et.al.00 (Bargiotti et al.), HLLL00 (Hoecker,Laplace,Lacker,LeDiberder), M01 (Mele), UTFit (Bona et al.). CKMFitter (J.Charles et al.). The full (dotted) lines correspond to the 68\%(95\%) probability regions. The star (for year '06) corresponds to the recent measured value by CDF [9]. The error of the experimental measurement cannot be appreciated with this scale.

- The fit prediction for $\sin 2 \beta$ is less precise than the fit result, so that the direct measurement is still dominant. However, there is a tension between the two determinations. As can be seen from the compatibility plot in Fig. 4 , the deviation of the measured $\sin 2 \beta$ from the fit prediction is below $2 \sigma$.

- The same happens for $\left|V_{u b}\right|$. In this case, it is interesting to consider separately the compatibility of the inclusive and exclusive measurements with the fit prediction. It turns out that the exclusive determination, based on Lattice QCD, is fully compatible with the fit prediction, while the more precise inclusive determination deviates by less than $2 \sigma$.

The redundance of the fit suggests that the UT analysis can be generalized to constrain possible NP contributions in the flavour sector. Indeed, it turns out that very stringent constraints on NP 


\begin{tabular}{ccccc}
\hline Parameter & \multicolumn{2}{c}{ Global fit } & \multicolumn{2}{c}{ Fit prediction } \\
& $68 \%$ & $95 \%$ & $68 \%$ & $95 \%$ \\
$\bar{\rho}$ & $0.154 \pm 0.022$ & {$[0.110,0.198]$} & - & - \\
$\bar{\eta}$ & $0.342 \pm 0.014$ & {$[0.315,0.371]$} & - & - \\
$\alpha\left[^{\circ}\right]$ & $92.0 \pm 3.4$ & {$[85.1,99.2]$} & $92.4 \pm 4.1$ & {$[84.1,100.5]$} \\
$\beta\left[^{\circ}\right]$ & $22.0 \pm 0.8$ & {$[20.5,23.7]$} & - & - \\
$\gamma\left[^{\circ}\right]$ & $65.6 \pm 3.3$ & {$[58.7,72.5]$} & $64.2 \pm 3.1$ & {$[56.7,71.1]$} \\
$\sin 2 \beta$ & $0.695 \pm 0.020$ & {$[0.656,0.736]$} & $0.736 \pm 0.034$ & {$[0.672,0.802]$} \\
$\left|V_{u b}\right|\left[10^{-4}\right]$ & $36.0 \pm 1.2$ & {$[33.7,38.6]$} & $34.8 \pm 1.6$ & {$[31.9,38.0]$} \\
$\Delta m_{s}\left[\mathrm{ps}^{-1}\right]$ & $17.7 \pm 0.1$ & {$[17.4,18.0]$} & $16.8 \pm 1.6$ & {$[13.8,20.4]$} \\
$\sin 2 \beta_{s}$ & $0.0366 \pm 0.0015$ & {$[0.0336,0.0397]$} & - & - \\
\hline
\end{tabular}

Table 1: Values and probability ranges for the UT parameters obtained from the UT fit. Second and third column (Global Fit): results obtained using all constraints. Fourth and fifth column (Fit Predictions): for those parameters for which an experimental determination is available, results obtained without using the experimental determination.

contributions to meson mixing amplitudes can be obtained, allowing us to probe energies much higher than the electroweak scale. We discuss this in detail in Section 4. Before presenting results for NP, in Section 3 we discuss the role of lattice QCD in the SM analysis. Finally, in 5 we discuss future perspectives for NP analyses with improved experimental and lattice QCD inputs.

\section{Lattice QCD and the SM Unitarity Triangle Analysis}

Given the redundancy of the SM UT fit, assuming the validity of the SM it is possible to perform an "experimental" determination of several hadronic quantities which were previously taken from lattice QCD calculations. This approach has two important advantages. The first one is that we have the possibility of making a full UT analysis without relying at all on theoretical calculations of hadronic matrix elements, for which there was a long debate about the treatment of values and error distributions. The second advantage is that we can extract from the combined experimental measurements the value of $\hat{B}_{K}$ and of the $B^{0}$ mixing amplitudes $f_{B_{s, d}} \hat{B}_{B_{s, d}}^{1 / 2}$ (or equivalently $f_{B_{s}} \hat{B}_{B_{s}}^{1 / 2}$ and $\xi$ ) and compare them to the theoretical predictions.

In Tab. 2 we give the results for $\hat{B}_{K}, f_{B_{s}} \hat{B}_{B_{s}}^{1 / 2}$ and $\xi$ obtained using the information from the UT angles plus $\left|V_{u b} / V_{c b}\right|$ measurements. We also give the values of $f_{B}$ obtained from this fit, using in addition the lattice value of $\hat{B}_{B_{d}}$. In the last column of the table we give the lattice values used as input by the UTfit Collaboration [24] for an easier comparison with those extracted from the UT fit. See ref. [25] for a compilation of recent lattice results.

Clearly the agreement between the "experimental" and Lattice QCD determinations of the parameters in Table 2 is excellent. By comparing the error of the two determinations in Table 2, we see that Lattice input in the SM UTA is important for $B_{K}$ and $\xi$, while the fit determines $f_{B_{s}} \hat{B}_{B_{s}}^{1 / 2}$ and $f_{B}$ much better than Lattice. 


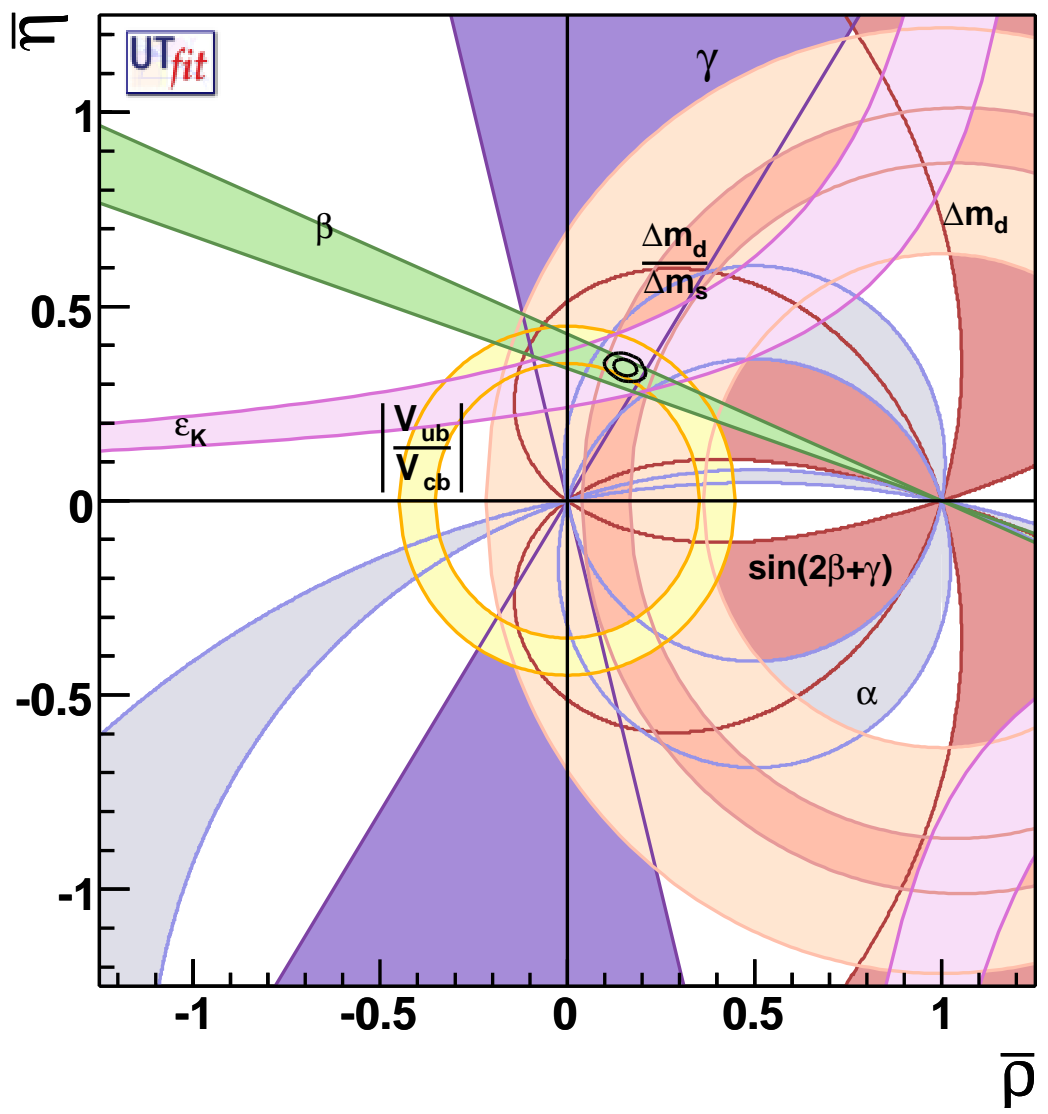

Figure 2: Determination of $\bar{\rho}$ and $\bar{\eta}$ from constraints on $\left|V_{u b}\right| /\left|V_{c b}\right|, \Delta m_{d}, \Delta m_{s}, \varepsilon_{k}, \beta, \gamma$, and $\alpha$. 68\% and $95 \%$ total probability contours are shown, together with $95 \%$ probability regions from the individual constraints.

In Figs. 6 we show the allowed probability regions in the $f_{B_{s}} \hat{B}_{B_{s}}^{1 / 2}$ vs. $\xi, f_{B_{s}} \hat{B}_{B_{s}}^{1 / 2}$ vs. $B_{K}$ and $B_{K}$ vs. $\xi$ planes.

The phenomenological extraction of the hadronic parameters and the comparison with lattice results assumes the validity of the SM and it is meaningful in this framework only. A similar strategy could be followed in any given extension of the SM when enough experimental information is available. In general, however, a model-independent UT analysis beyond the SM cannot be carried out without some "a priori" theoretical knowledge of the relevant hadronic parameters. For this reason the error in the calculation of the hadronic matrix elements affects the uncertainties in the determination of the NP parameters that is the topic of the next Section.

\section{UT fit beyond the SM and searches for NP}

Our aim in this Section is to consider the most general effective Hamiltonian for $\Delta F=2$ processes $\left(\mathscr{H}_{\Delta F=2}^{\text {eff }}\right)$ and to translate the experimental constraints into allowed ranges for the Wilson 

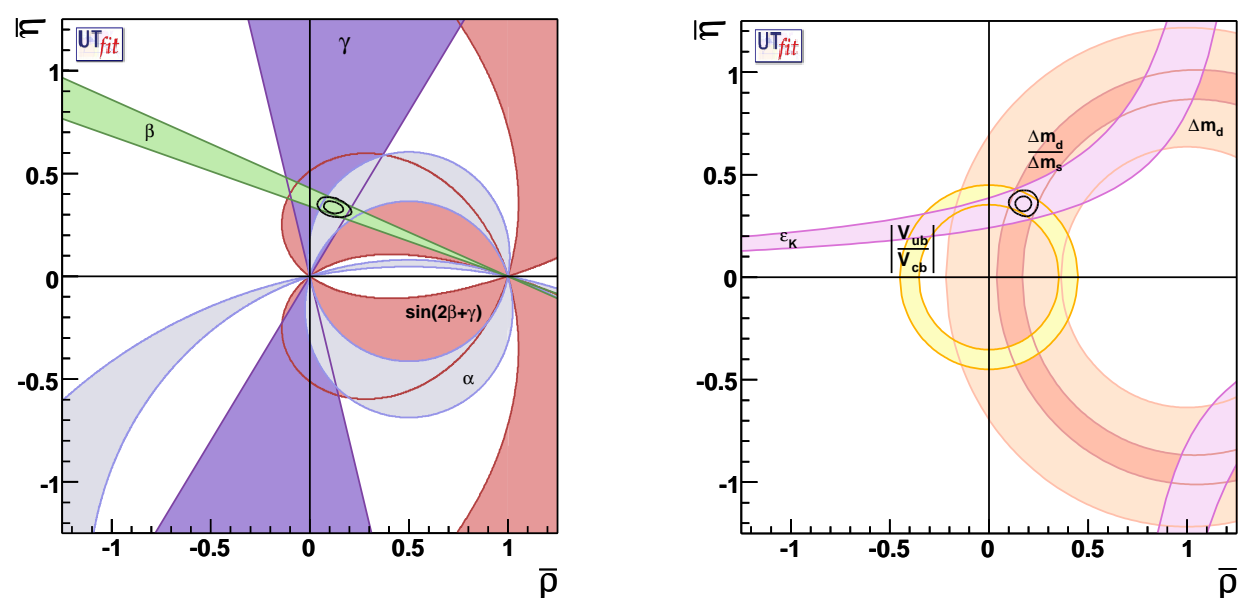

Figure 3: Left panel: determination of $\bar{\rho}$ and $\bar{\eta}$ from constraints on $\beta, \gamma$ and $\alpha$. Right panel: determination of $\bar{\rho}$ and $\bar{\eta}$ from constraints on $\left|V_{u b}\right| /\left|V_{c b}\right|, \Delta m_{d}, \Delta m_{s}$ and $\varepsilon_{k}$. 68\% and $95 \%$ total probability contours are shown, together with $95 \%$ probability regions from the individual constraints.
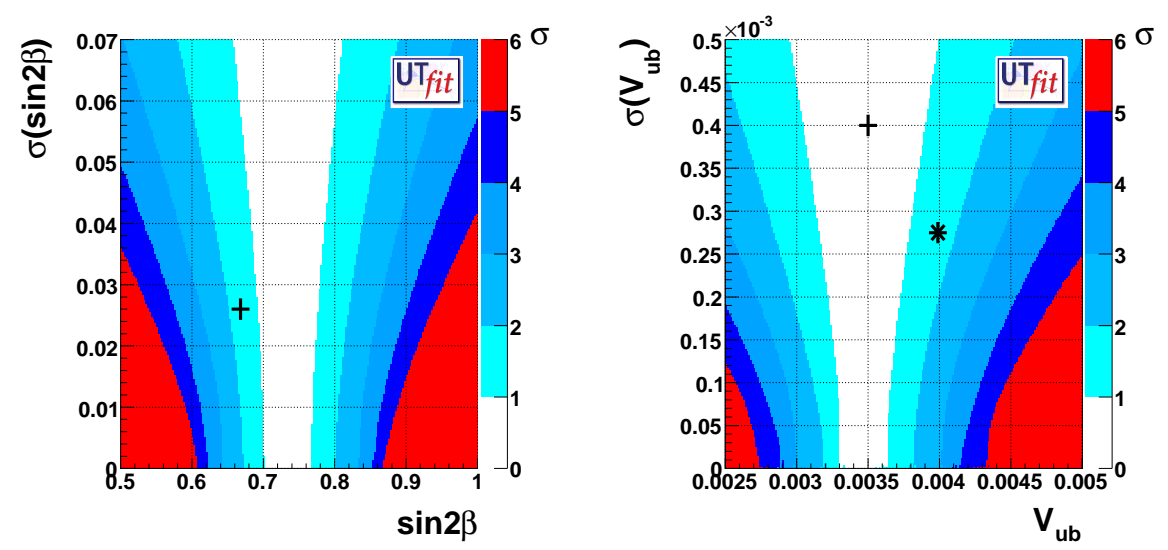

Figure 4: Compatibility plots for $\sin 2 \beta$ (left) and $\left|V_{u b}\right|$ (right).

coefficients of $\mathscr{H}_{\Delta F=2}^{\text {eff }}$. These coefficients in general have the form

$$
C_{i}(\Lambda)=F_{i} \frac{L_{i}}{\Lambda^{2}}
$$

where $F_{i}$ is a function of the (complex) NP flavour couplings, $L_{i}$ is a loop factor that is present in models with no tree-level Flavour Changing Neutral Currents (FCNC), and $\Lambda$ is the scale of NP, i.e. the typical mass of the new particles mediating the $\Delta F=2$ transition. For a generic stronglyinteracting theory with arbitrary flavour structure, we expect $F_{i} \sim L_{i} \sim 1$ so that the allowed range for each of the $C_{i}(\Lambda)$ can be immediately translated into a lower bound on $\Lambda$. Specific assumptions on the flavour structure of NP, for example Minimal or Next-to-Minimal Flavour Violation (MFV or NMFV), correspond to particular choices of the $F_{i}$ functions, as detailed below. 


\begin{tabular}{ccc}
\hline \hline Parameter & UT angles $+\left|V_{u b} / V_{c b}\right|$ & Lattice \\
\hline$\hat{B}_{K}$ & $0.74 \pm 0.07$ & $0.75 \pm 0.07$ \\
\hline$f_{B_{s}} \hat{B}_{B_{s}}^{1 / 2}(\mathrm{MeV})$ & $264.7 \pm 3.6$ & $270 \pm 30$ \\
\hline$\xi$ & $1.26 \pm 0.05$ & $1.21 \pm 0.04$ \\
\hline$f_{B}(\mathrm{MeV})$ & $191 \pm 13$ & $200 \pm 20$ \\
\hline \hline
\end{tabular}

Table 2: Comparison of determinations of the hadronic parameters from the constraints on the angles $\alpha, \beta$, and $\gamma$ and from $\left|V_{u b} / V_{c b}\right|$ with the Lattice QCD averages from ref. [24] (Lattice).
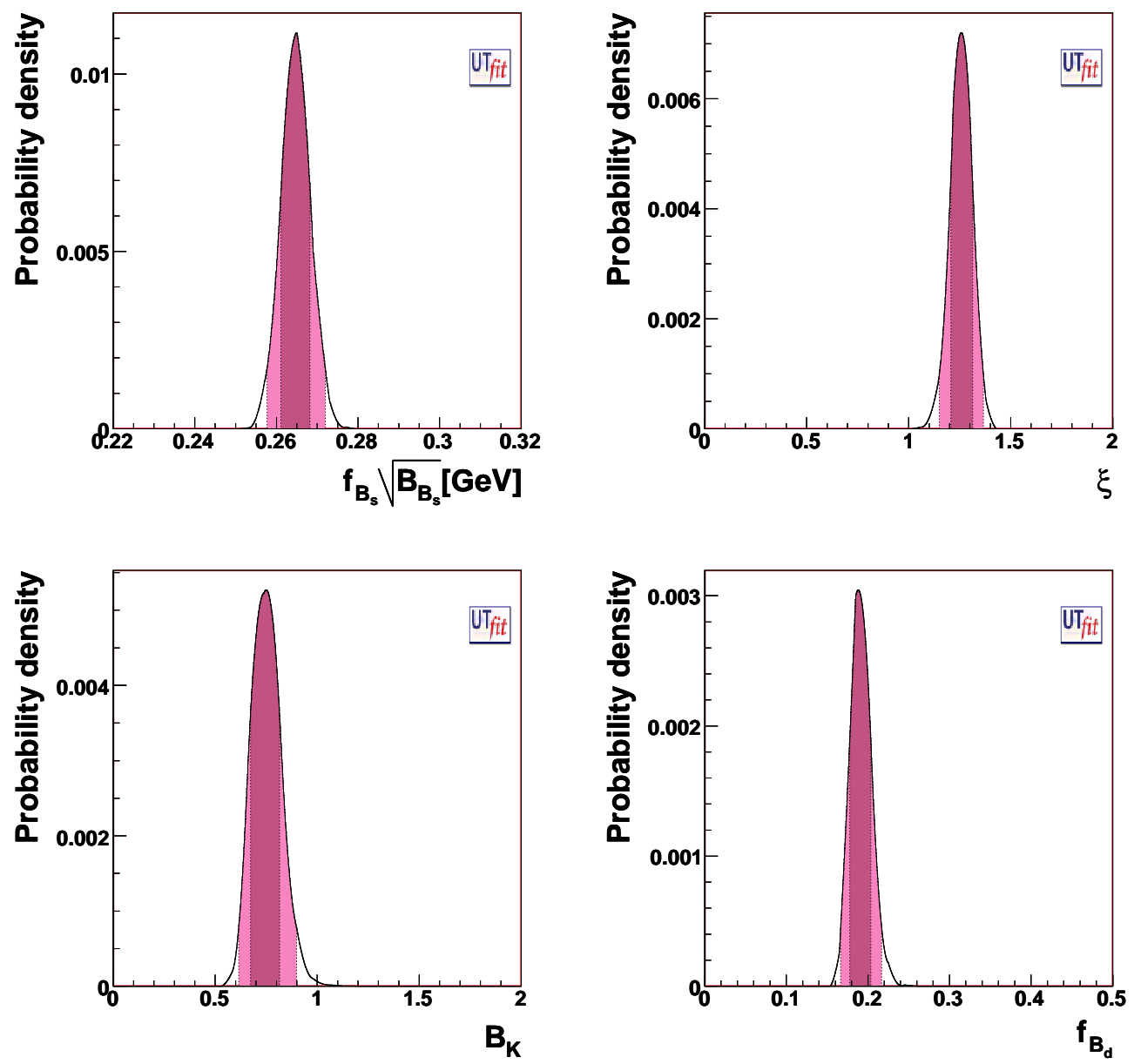

Figure 5: Determination of $f_{B_{s}} \sqrt{\hat{B}_{s}}$ (top-left), $\xi$ (top-right), $\hat{B}_{K}$ (bottom-left) and $f_{B}$ (bottom-right) obtained from the UT angles fit.

This approach is analogous to the operator analysis of electroweak precision observables performed in [26], but it provides much more stringent bounds on models with nonminimal flavour violation. In particular, we find that the scale of heavy particles mediating tree-level FCNC in models of NMFV must be above $12 \mathrm{TeV}$, making them undetectable at the LHC. This bound applies for 

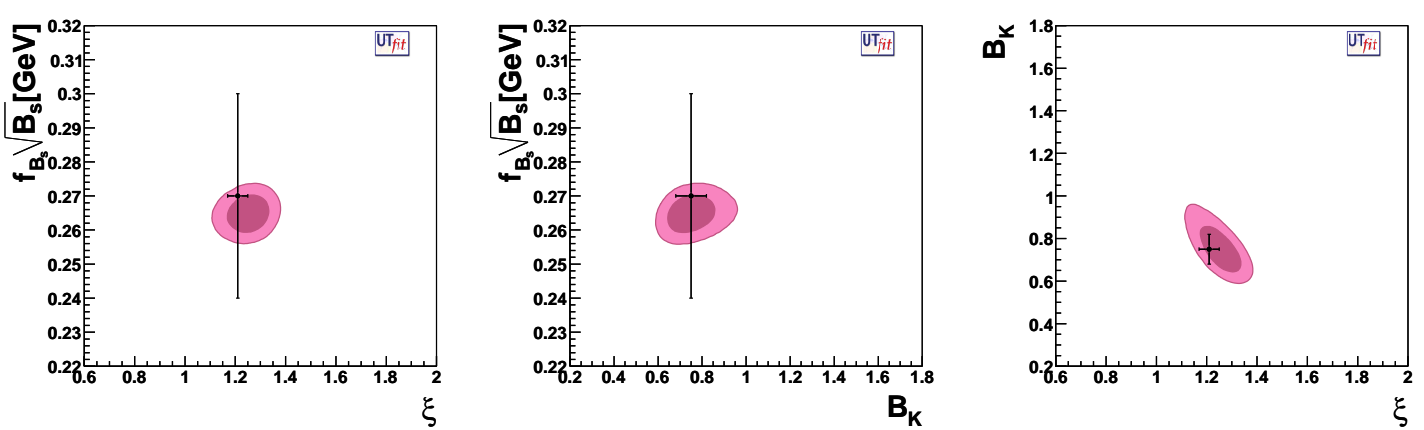

Figure 6: Constraints in the $f_{B_{s}} \sqrt{\hat{B}_{s}}$ vs. $\xi, f_{B_{s}} \sqrt{\hat{B}_{s}}$ vs. $B_{K}$ and $B_{K}$ vs. $\xi$ planes. The error bars show the results from lattice $\mathrm{QCD}$ calculations.

instance to the Kaluza-Klein excitations of gauge bosons in a large class of models with (warped) extra dimensions [27]. Flavour physics remains the main avenue to probe such extensions of the SM.

The contribution of NP to $\Delta F=2$ transitions can be parameterized in a model-independent way as the ratio of the full (SM+NP) amplitude to the SM one. In this way, we can define the parameters $C_{B_{q}}$ and $\phi_{B_{q}}(q=d, s)$ as [28]:

$$
C_{B_{q}} e^{2 i \phi_{B_{q}}}=\frac{\left\langle B_{q}\left|H_{\mathrm{eff}}^{\mathrm{full}}\right| \bar{B}_{q}\right\rangle}{\left\langle B_{q}\left|H_{\mathrm{eff}}^{\mathrm{SM}}\right| \bar{B}_{q}\right\rangle}=\frac{A_{q}^{\mathrm{SM}} e^{2 i \phi_{q}^{\mathrm{SM}}}+A_{q}^{\mathrm{NP}} e^{2 i\left(\phi_{q}^{\mathrm{SM}}+\phi_{q}^{\mathrm{NP}}\right)}}{A_{q}^{\mathrm{SM}} e^{2 i \phi_{q}^{\mathrm{SM}}}},
$$

and write all the measured observables as a function of these parameters and the SM ones $(\bar{\rho}, \bar{\eta}$, and additional parameters such as masses, form factors, and decay constants). Details are given in ref. [29, 30, 31, 32]. In a similar way, one can write

$$
C_{\varepsilon_{K}}=\frac{\operatorname{Im}\left[\left\langle K^{0}\left|H_{\text {eff }}^{\mathrm{full}}\right| \bar{K}^{0}\right\rangle\right]}{\operatorname{Im}\left[\left\langle K^{0}\left|H_{\mathrm{eff}}^{\mathrm{SM}}\right| \bar{K}^{0}\right\rangle\right]}, \quad C_{\Delta m_{K}}=\frac{\operatorname{Re}\left[\left\langle K^{0}\left|H_{\mathrm{eff}}^{\mathrm{full}}\right| \bar{K}^{0}\right\rangle\right]}{\operatorname{Re}\left[\left\langle K^{0}\left|H_{\mathrm{eff}}^{\mathrm{SM}}\right| \bar{K}^{0}\right\rangle\right]} .
$$

Concerning $\Delta m_{K}$, to be conservative we add to the short-distance contribution a possible longdistance one that varies with a uniform distribution between zero and the experimental value of $\Delta m_{K}$.

We perform a global analysis and determine simultaneously $\bar{\rho}, \bar{\eta}, C_{B_{q}}, \phi_{B_{q}}, C_{\mathcal{E}_{K}}$ and $C_{\Delta m_{K}}$. The resulting probability density function (p.d.f.) in the $\bar{\rho}-\bar{\eta}$ plane is shown in Fig. 7. Even at $95 \%$ probability, only a small region close to the SM fit result survives. The results for $\bar{\rho}$ and $\bar{\eta}$ reported in Tab. 3 are at a level of accuracy comparable to the SM fit in Tab. 1, so that the SM contribution to FCNC processes in the presence of arbitrary NP is bound to lie very close to the results of the SM in the absence of NP. This result represents a major improvement in the study of FCNC processes beyond the SM, and opens up the possibility of precision studies of flavour processes in the presence of NP.

The constraining power of this analysis is evident in the results for the NP parameters given in Tab. 3 and shown in Fig. 8. The most striking feature in Fig. 8 is that, while $K-\bar{K}$ and $B_{d}-\bar{B}_{d}$ mixings show a remarkable agreement with the SM, the phase of the $B_{S}-\bar{B}_{s}$ mixing amplitude 
appears to be far away from the SM prediction. Indeed, we find that the SM value $\phi_{B_{s}}=0$ is included only in the $99.6 \%$ probability range, corresponding to $2.9 \sigma$. Thus, NP contributions to $B_{s}-\bar{B}_{s}$ mixing carrying a new CP-violating phase seem to be comparable to, if not dominant on, the SM amplitude, disfavouring the SM as well as models of MFV. This can be seen in Fig. 9, where we report the $68 \%$ and $95 \%$ probability regions in the planes $A_{\mathrm{NP}} / A_{\mathrm{SM}} \mathrm{vs} \phi_{\mathrm{NP}}$ for $B_{d}$ and $B_{s}$ mixings. The pattern of NP contributions emerging from Figs. 8 and 9 is the following:

- NP effects in $\varepsilon_{K}$ and $B_{d}$ mixing are possible at a level $\lesssim 20-30 \%$;

- There is evidence for new CP-violating effects in $B_{s}$ mixing with an amplitude comparable to the $\mathrm{SM}$ one and a large $\mathrm{CP}$-violating phase $[33,32]$.

Thus, a peculiar NP flavour structure emerges from the requirement of having large new CPviolating effects in $B_{s}$ mixing but not in $B_{d}$ and $K$ mixings. This structure is however theoretically well-motivated, both in nonabelian flavour models and in SUSY-GUTs, where neutrino oscillations can be connected to large flavour violation in $b \leftrightarrow s$ transitions [34].

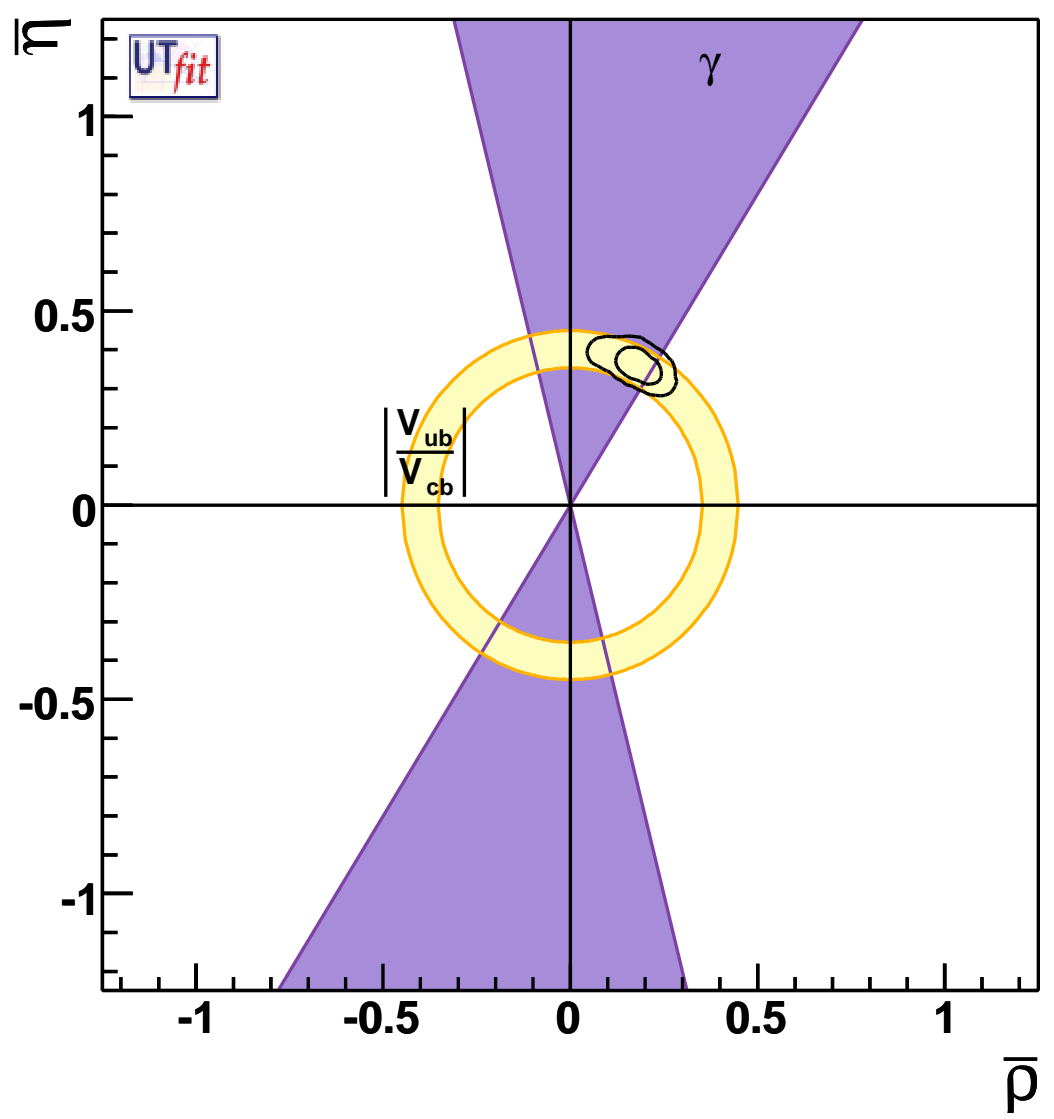

Figure 7: Determination of $\bar{\rho}$ and $\bar{\eta}$ from the NP generalized analysis. $68 \%$ and $95 \%$ probability regions for $\bar{\rho}$ and $\bar{\eta}$ are shown, together with the $1 \sigma$ contours given by the tree-level determination of $\left|V_{u b}\right|$ and $\gamma$. 


\begin{tabular}{cccccc}
\hline \hline Parameter & Output & Parameter & Output & Parameter & Output \\
\hline \hline$C_{B_{d}}$ & $0.96 \pm 0.23$ & $\phi_{B_{d}}\left[^{\circ}\right]$ & $-2.9 \pm 1.9$ & $C_{B_{s}}$ & $0.97 \pm 0.20$ \\
\hline$\phi_{B_{s}}\left[^{\circ}\right]$ & $-70 \pm 7 \cup-18 \pm 7$ & $C_{\varepsilon_{K}}$ & $0.99 \pm 0.16$ & & \\
\hline \hline $\bar{\rho}$ & $0.177 \pm 0.044$ & $\bar{\eta}$ & $0.360 \pm 0.031$ & $\alpha\left[^{\circ}\right]$ & $92 \pm 7$ \\
\hline$\gamma\left[^{\circ}\right]$ & $63 \pm 7$ & $\sin 2 \beta$ & $0.734 \pm 0.038$ & $\sin 2 \beta_{s}$ & $0.038 \pm 0.003$ \\
\hline \hline
\end{tabular}

Table 3: Determination of UT and NP parameters from the UT fit.
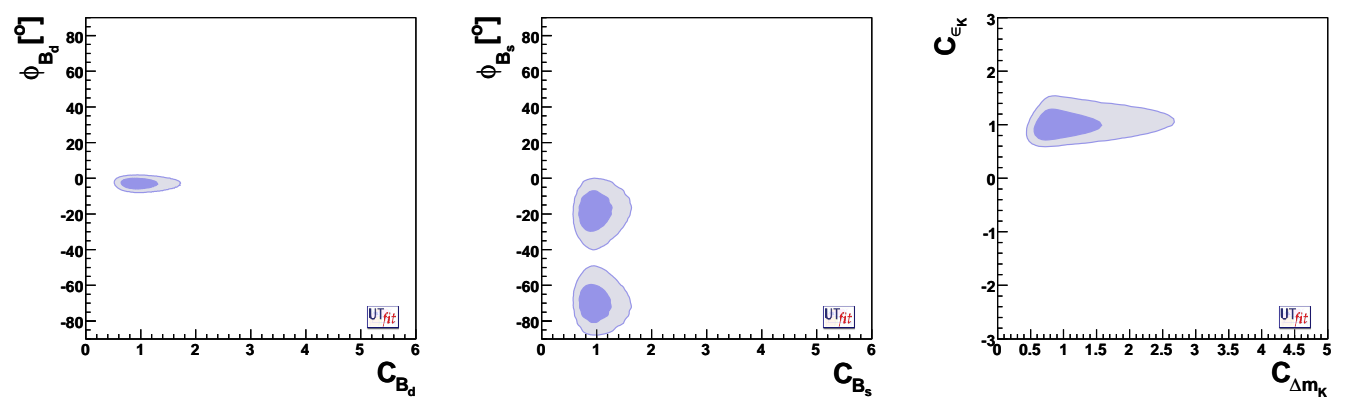

Figure 8: From left to right, constraints on $\phi_{B_{d}}$ vs. $C_{B_{d}}, \phi_{B_{s}}$ vs. $C_{B_{s}}$ and $C_{\varepsilon_{K}}$ vs $C_{\Delta m_{K}}$ from the NP generalized analysis.
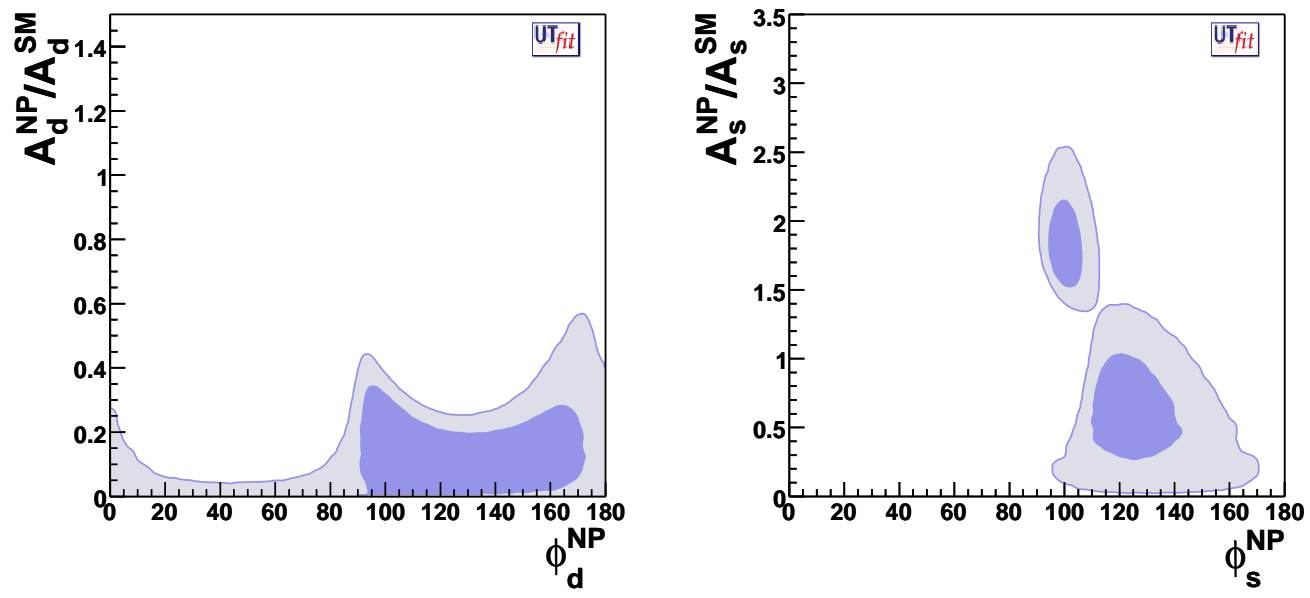

Figure 9: $68 \%$ and $95 \%$ probability regions in the planes $A_{\mathrm{NP}} / A_{\mathrm{SM}}$ vs $\phi_{\mathrm{NP}}$ for $B_{d}$ (left) and $B_{s}$ (right) mixings.

It is important to stress that the information contained in these constraints does not rely on any specific model for NP. The list of applications in NP phenomenology is rich. For instance, restricting to the case of SUSY models, the two-dimensional constraint of the $b \rightarrow s(b \rightarrow d)$ sector can be translated into a limit on the mass-insertion complex parameters $\delta_{23}^{d}\left(\delta_{13}^{d}\right)$, using the NLO supersymmetric expression of the $B_{q}-\bar{B}_{q}$ mixing amplitude [15]. This bound, combined with the 
constraint from $b \rightarrow s$ decays [35], allows to obtain the best available information on the offdiagonal terms of the squark mass matrix. However, in the following, rather than considering an explicit model for NP, we perform a general analysis based on the most general Hamiltonian for $\Delta F=2$ processes.

The most general effective Hamiltonians for $\Delta F=2$ processes beyond the SM have the following form:

$$
\begin{aligned}
\mathscr{H}_{\mathrm{eff}}^{K-\bar{K}} & =\sum_{i=1}^{5} C_{i} Q_{i}^{s d}+\sum_{i=1}^{3} \tilde{C}_{i} \tilde{Q}_{i}^{s d} \\
\mathscr{H}_{\mathrm{eff}}^{B_{q}-\bar{B}_{q}} & =\sum_{i=1}^{5} C_{i} Q_{i}^{b q}+\sum_{i=1}^{3} \tilde{C}_{i} \tilde{Q}_{i}^{b q}
\end{aligned}
$$

where

$$
\begin{aligned}
& Q_{1}^{q_{i} q_{j}}=\bar{q}_{j L}^{\alpha} \gamma_{\mu} q_{i L}^{\alpha} \bar{q}_{j L}^{\beta} \gamma^{\mu} q_{i L}^{\beta}, \\
& Q_{2}^{q_{i} q_{j}}=\bar{q}_{j R}^{\alpha} q_{i L}^{\alpha} \bar{q}_{j R}^{\beta} q_{i L}^{\beta}, \\
& Q_{3}^{q_{i} q_{j}}=\bar{q}_{j R}^{\alpha} q_{i L}^{\beta} \bar{q}_{j R}^{\beta} q_{i L}^{\alpha}, \\
& Q_{4}^{q_{i} q_{j}}=\bar{q}_{j R}^{\alpha} q_{i L}^{\alpha} \bar{q}_{j L}^{\beta} q_{i R}^{\beta}, \\
& Q_{5}^{q_{i} q_{j}}=\bar{q}_{j R}^{\alpha} q_{i L}^{\beta} \bar{q}_{j L}^{\beta} q_{i R}^{\alpha},
\end{aligned}
$$

and the operators $\tilde{Q}_{1,2,3}^{q_{i} q_{j}}$ are obtained from the $Q_{1,2,3}^{q_{i} q_{j}}$ by the exchange $L \leftrightarrow R$. Here $q_{R, L}=P_{R, L} q$, with $P_{R, L}=\left(1 \pm \gamma_{5}\right) / 2$, and $\alpha$ and $\beta$ are colour indices.

The NLO anomalous dimension matrix has been computed in [12]. We use the RegularisationIndependent anomalous dimension matrix in the Landau gauge (also known as RI-MOM).

The $C_{i}(\Lambda)$ are obtained by integrating out all new particles simultaneously at the NP scale $\Lambda$. We then have to evolve the coefficients down to the hadronic scales $\mu_{b}=m_{b}=4.6 \mathrm{GeV}\left(m_{b} \equiv\right.$ $m_{b}\left(\mu=m_{b}\right)$ is the RI-MOM mass) for bottom mesons and $\mu_{K}=2 \mathrm{GeV}$ for Kaons, which are the renormalisation scales of the operators used in lattice computations for the matrix elements.

Using the analytic formulae given in ref. [31] and the matrix elements given in ref. [24], we switch on one coefficient at a time in each sector and calculate its value from the result of the NP analysis presented above. In this way, we obtain the p.d.f. for the Wilson coefficients at the NP scale $\Lambda$.

The connection between the $C_{i}(\Lambda)$ and the NP scale $\Lambda$ depends on the general properties of the NP model, and in particular on the flavour structure of the $F_{i}$. Assuming strongly interacting new particles, we have from eq. (4.1) with $L_{i}=1$

$$
\Lambda=\sqrt{\frac{F_{i}}{C_{i}}}
$$

Let us now discuss two notable examples:

- In the case of NMFV, we have $\left|F_{i}\right|=F_{\mathrm{SM}}$ with an arbitrary phase. This condition is realized in models in which right-handed currents also contribute to FCNC processes, but with the same hierarchical structure in the mixing angles as in the SM left-handed currents. Given the 
order-of-magnitude equalities $m_{d} / m_{b} \sim\left|V_{t d}\right|, m_{s} / m_{b} \sim\left|V_{t s}\right|$, bounds obtained in this scenario are also of interest for extra-dimensional models with FCNC couplings suppressed linearly with quark masses. Clearly, given the QCD and, for $K-\bar{K}$ mixing, chiral enhancement of NP operators, the constraints on the NP scale are much stronger for NMFV than for MFV.

- For arbitrary NP flavour structures, we expect $\left|F_{i}\right| \sim 1$ with arbitrary phase. In this case, the constraints on the NP scale are much tighter due to the absence of the CKM suppression in the NP contributions.

In deriving the lower bounds on the NP scale $\Lambda$, we assume $L_{i}=1$, corresponding to stronglyinteracting and/or tree-level NP. Two other interesting possibilities are given by loop-mediated NP contributions proportional to $\alpha_{s}^{2}$ or $\alpha_{W}^{2}$. The first case corresponds for example to gluino exchange in the MSSM. The second case applies to all models of SM-like loop-mediated weak interactions. To obtain the lower bound on $\Lambda$ for loop-mediated contributions, one simply multiplies the bounds we quote in the following by $\alpha_{s}(\Lambda) \sim 0.1$ or by $\alpha_{W} \sim 0.03$.

\begin{tabular}{cccc}
\hline \hline Parameter & $\begin{array}{c}95 \% \text { allowed range } \\
\left(\mathrm{GeV}^{-2}\right)\end{array}$ & $\begin{array}{c}\text { Lower limit on } \Lambda(\mathrm{TeV}) \\
\text { for arbitrary NP }\end{array}$ & $\begin{array}{c}\text { Lower limit on } \Lambda(\mathrm{TeV}) \\
\text { for NMFV }\end{array}$ \\
\hline $\operatorname{Re} C_{K}^{1}$ & {$[-9.6,9.6] \cdot 10^{-13}$} & $1.0 \cdot 10^{3}$ & 0.35 \\
$\operatorname{Re} C_{K}^{2}$ & {$[-1.8,1.9] \cdot 10^{-14}$} & $7.3 \cdot 10^{3}$ & 2.0 \\
$\operatorname{Re} C_{K}^{3}$ & {$[-6.0,5.6] \cdot 10^{-14}$} & $4.1 \cdot 10^{3}$ & 1.1 \\
$\operatorname{Re} C_{K}^{4}$ & {$[-3.6,3.6] \cdot 10^{-15}$} & $17 \cdot 10^{3}$ & 4.0 \\
$\operatorname{Re} C_{K}^{5}$ & {$[-1.0,1.0] \cdot 10^{-14}$} & $10 \cdot 10^{3}$ & 2.4 \\
\hline $\operatorname{Im} C_{K}^{1}$ & {$[-4.4,2.8] \cdot 10^{-15}$} & $1.5 \cdot 10^{4}$ & 5.6 \\
$\operatorname{Im} C_{K}^{2}$ & {$[-5.1,9.3] \cdot 10^{-17}$} & $10 \cdot 10^{4}$ & 28 \\
$\operatorname{Im} C_{K}^{3}$ & {$[-3.1,1.7] \cdot 10^{-16}$} & $5.7 \cdot 10^{4}$ & 19 \\
$\operatorname{Im} C_{K}^{4}$ & {$[-1.8,0.9] \cdot 10^{-17}$} & $24 \cdot 10^{4}$ & 62 \\
$\operatorname{Im} C_{K}^{5}$ & {$[-5.2,2.8] \cdot 10^{-17}$} & $14 \cdot 10^{4}$ & 37 \\
\hline \hline$\left|C_{D}^{1}\right|$ & $<7.2 \cdot 10^{-13}$ & $1.2 \cdot 10^{3}$ & 0.40 \\
$\left|C_{D}^{2}\right|$ & $<1.6 \cdot 10^{-13}$ & $2.5 \cdot 10^{3}$ & 0.82 \\
$\left|C_{D}^{3}\right|$ & $<3.9 \cdot 10^{-12}$ & $0.51 \cdot 10^{3}$ & 0.17 \\
$\left|C_{D}^{4}\right|$ & $<4.8 \cdot 10^{-14}$ & $4.6 \cdot 10^{3}$ & 1.5 \\
$\left|C_{D}^{5}\right|$ & $<4.8 \cdot 10^{-13}$ & $1.4 \cdot 10^{3}$ & 0.47 \\
\hline \hline$\left|C_{B_{d}}^{1}\right|$ & $<2.3 \cdot 10^{-11}$ & $0.21 \cdot 10^{3}$ & 1.8 \\
$\left|C_{B_{d}}^{2}\right|$ & $<7.2 \cdot 10^{-13}$ & $1.2 \cdot 10^{3}$ & 8.5 \\
$\left|C_{B_{d}}^{3}\right|$ & $<2.8 \cdot 10^{-12}$ & $0.60 \cdot 10^{3}$ & 4.4 \\
$\left|C_{B_{d}}^{4}\right|$ & $<2.1 \cdot 10^{-13}$ & $2.2 \cdot 10^{3}$ & 14 \\
$\left|C_{B_{d}}^{5}\right|$ & $<6.0 \cdot 10^{-13}$ & $1.3 \cdot 10^{3}$ & 8.8 \\
\hline \hline & & & \\
\hline
\end{tabular}

Table 4: $95 \%$ probability range for $C(\Lambda)$ and the corresponding lower bounds on the NP scale $\Lambda$ for arbitrary NP flavour structure and for NMFV. See the text for details.

Considering NP contributions to $K, D$ and $B_{d}$ mixing, ref. [31] obtains the $95 \%$ probability 
upper bounds on the absolute value of $C^{i}$ reported in Tab. 4. This result is completely modelindependent.

Assuming strongly interacting and/or tree-level NP contributions with generic flavour structure (i.e. $L_{i}=\left|F_{i}\right|=1$ ), we can translate the upper bounds on $C_{i}$ into the lower bounds on the NP scale $\Lambda$ reported in the third column of Tab. 4. As anticipated above, we see that in the $K^{0}$ sector all bounds from non-standard operators are one order of magnitude stronger than the bound from the SM operator, due to the chiral enhancement. In addition, operator $Q_{4}$ has the strongest Renormalization Group (RG) enhancement. In the $D^{0}$ and $B_{d}$ sectors, the chiral enhancement is absent, but the RG enhancement is still effective. The overall constraint on the NP scale $\Lambda$ comes from $\operatorname{Im} C_{K}^{4}$ and reads, for strongly interacting and/or tree-level NP, $\alpha_{s}$ loop mediated or $\alpha_{W}$ loop mediated respectively:

$$
\Lambda_{\text {tree }}^{\mathrm{GEN}}>2.4 \cdot 10^{5} \mathrm{TeV}, \quad \Lambda_{\alpha_{\mathrm{s}}}^{\mathrm{GEN}}>2.4 \cdot 10^{4} \mathrm{TeV}, \quad \Lambda_{\alpha_{\mathrm{W}}}^{\mathrm{GEN}}>8 \cdot 10^{3} \mathrm{TeV} .
$$

Assuming strongly interacting and/or tree-level NP contributions with NMFV flavour structure (i.e. $L_{i}=1$ and $\left|F_{i}\right|=\left|F_{\mathrm{SM}}\right|$ ), we can translate the upper bounds on $C_{i}$ into the lower bounds on the NP scale $\Lambda$ reported in the fourth column of Tab. 4 . The flavour structure of NMFV models implies that the bounds from the four sectors are all comparable, the strongest one being obtained from $\operatorname{Im} C_{K}^{4}$ (barring, as always, accidental cancellations):

$$
\Lambda_{\text {tree }}^{\mathrm{NMFV}}>62 \mathrm{TeV}, \quad \Lambda_{\alpha_{\mathrm{s}}}^{\mathrm{NMFV}}>6.2 \mathrm{TeV}, \quad \Lambda_{\alpha_{\mathrm{W}}}^{\mathrm{NMFV}}>2 \mathrm{TeV} .
$$

Conversely, the NP evidence in $B_{s}$ mixing can be converted into an upper bound on the NP scale for a given flavour structure. This upper bound can be compared with the lower bound from the other sectors to assess the compatibility of experimental data with the flavour structure of the NP model under consideration.

\section{Future prospects}

To conclude this talk, let us discuss the prospects for flavour physics until the year 2015, including future developments both in Lattice QCD and in experiment, with LHCb and, hopefully, SuperB [36]. The goal is to bring the accuracy of the determination of SM and NP contributions to $\Delta F=2$ and $\Delta F=1$ processes to the percent level. Based on the discussion in Sec. 4 , this will allow us to probe scales at least a factor of three higher than what can be achieved today.

This ambitious goal requires big efforts from the Lattice QCD community. According to the SuperB CDR [36], reaching the required accuracy in 2015 is feasible even in the conservative scenario in which no improvements in algorithms are foreseen, just based on the increase in computing power. In Tab. 5 we report the projected accuracy on several key Lattice QCD results [36]. It is important to notice that the accuracy required should be reached not only for the matrix elements of SM operators, but also for the matrix elements of new operators arising in extensions of the SM.

In Fig. 10 we show the regions on the $\bar{\rho}-\bar{\eta}$ plane selected by different constraints assuming the current measurement precision, and that expected in 2015. With the precision reached in 2015, the current discrepancies would clearly indicate the presence of New Physics in the flavour sector!

Let us now discuss the accuracy of the UTA in 2015 both within the SM and in the presence of NP. Assuming the validity of the SM, we expect to be able to select a region in the $\bar{\rho}-\bar{\eta}$ plane as shown in Fig. 11. The corresponding numerical results are given in Table 6. 


\begin{tabular}{lccccc}
\hline \hline Measurement & $\begin{array}{c}\text { Hadronic } \\
\text { Parameter }\end{array}$ & $\begin{array}{c}\text { Present } \\
\text { Error }\end{array}$ & 6 TFlops & 60 TFlops & $\begin{array}{c}\text { 1-10 PFlops } \\
\text { (Year 2015) }\end{array}$ \\
\hline$K \rightarrow \pi l v$ & $f_{+}^{K \pi}(0)$ & $0.9 \%$ & $0.7 \%$ & $0.4 \%$ & $<0.1 \%$ \\
$\varepsilon_{K}$ & $\hat{B}_{K}$ & $11 \%$ & $5 \%$ & $3 \%$ & $1 \%$ \\
$B \rightarrow l v$ & $f_{B}$ & $14 \%$ & $3.5-4.5 \%$ & $2.5-4.0 \%$ & $1.0-1.5 \%$ \\
$\Delta m_{s}$ & $f_{B s} \sqrt{B_{B_{s}}}$ & $13 \%$ & $4-5 \%$ & $3-4 \%$ & $1-1.5 \%$ \\
$\Delta m_{d} / \Delta m_{s}$ & $\xi$ & $5 \%$ & $3 \%$ & $1.5-2 \%$ & $0.5-0.8 \%$ \\
$B \rightarrow D / D^{*} l v$ & $\mathscr{F}_{B \rightarrow D / D^{*}}$ & $4 \%$ & $2 \%$ & $1.2 \%$ & $0.5 \%$ \\
$B \rightarrow \pi / \rho l v$ & $f_{+}^{B \pi}, \ldots$ & $11 \%$ & $5.5-6.5 \%$ & $4-5 \%$ & $2-3 \%$ \\
$B \rightarrow K^{*} / \rho\left(\gamma, l^{+} l^{-}\right)$ & $T_{1}^{B \rightarrow K^{*} / \rho}$ & $13 \%$ & - & - & $3-4 \%$ \\
\hline
\end{tabular}

Table 5: Prediction of the accuracy that can be reached in lattice QCD determinations of various hadronic parameters assuming the availability of a computational power of about 6 TFlops (4th column), 60 TFlops (5th column) and 1-10 PFlops (6th column), from ref. [36]. The predictions given for the 6 TFlops and 60 TFlops cases have been presented by S. Sharpe in [37]. The accuracy reached at present in the determination of the various parameters is also shown (3rd column).
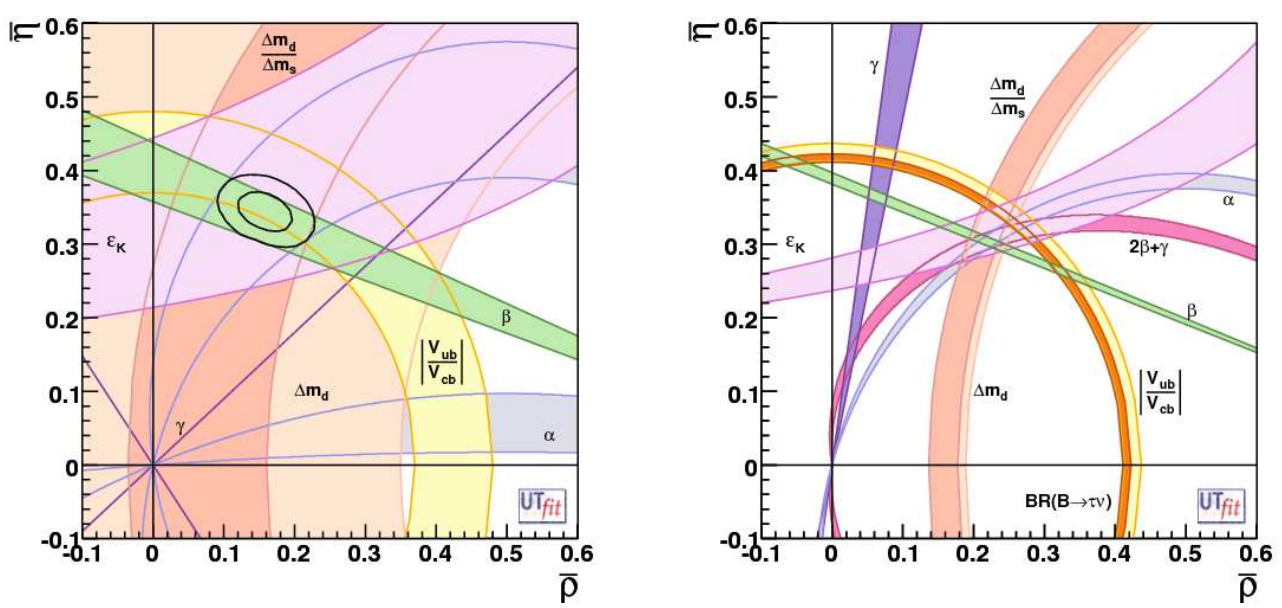

Figure 10: Regions corresponding to 95\% probability for $\bar{\rho}$ and $\bar{\eta}$ selected by different constraints, assuming present central values with present errors (left) or with errors expected in 2015 (right).

The results shown in Fig. 11 and in Table 6 indicate that a precision of a fraction of a percent can be reached, significantly improving the current situation, and providing a generic test of the presence of New Physics at that level of precision.

Moving to the NP generalized analysis, along the lines of the discussion in Sec. 4, ref. [36] obtains the results given in Table 6. The precision of the CKM parameters obtained in the presence of generic New Physics is not drastically worse than that of the Standard Model fit, and remains at the subpercent level. This is a good starting point for New Physics analyses, which require the model-independent determination of the CKM parameters as an input.

The fit using $\Delta F=2$ amplitudes with generic New Physics contributions also allows us to 


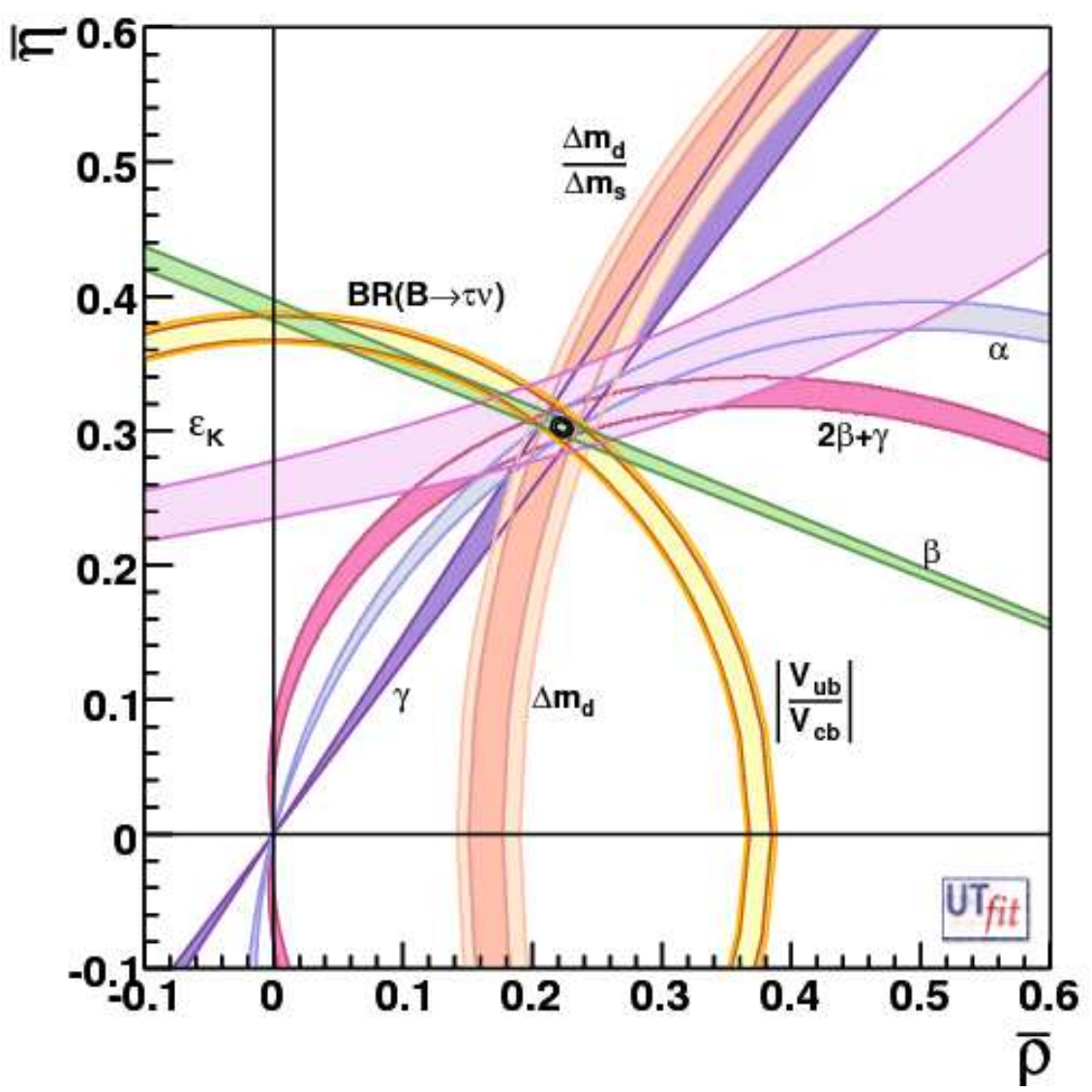

Figure 11: Allowed regions for $\bar{\rho}$ and $\bar{\eta}$ in 2015 assuming the validity of the SM, from ref. [36]. The closed contours at $68 \%$ and $95 \%$ probability are shown. The full lines correspond to $95 \%$ probability regions for each of the constraints.

obtain constraints on the New Physics parameters $C_{B_{d}}$ and $\phi_{B_{d}}$, which in turn provide information on the extent to which the experimental data allow for New Physics in $\Delta F=2$ amplitudes as discussed in Sec. 4. The numerical results from ref. [36] are given in Table 7. To illustrate the expected improvement in 2015, in Fig. 12 we show the allowed regions in the $C_{B_{d}}-\phi_{B_{d}}$ plane, as compared to the current situation, from ref. [36].

It is important to note that the reduction of the uncertainty on the parameter $C_{B_{d}}$ is largely due to the expected improvement of lattice calculations. By contrast, the impressive progress in the determination of $\phi_{B_{d}}$ is due to the improved precision of the experimental quantities measured at SuperB.

\section{Conclusions}

Lattice QCD will play a central role in the next decade. If new particles will be observed at the LHC, Lattice QCD will be crucial to determine the flavour structure of NP. If no new particles will 


\begin{tabular}{lcccc}
\hline \hline Parameter & SM Fit today & SM Fit in 2015 & New Physics fit today & New Physics fit in 2015 \\
\hline $\bar{\rho}$ & $0.154 \pm 0.022$ & \pm 0.0028 & $0.177 \pm 0.044$ & \pm 0.005 \\
$\bar{\eta}$ & $0.342 \pm 0.014$ & \pm 0.0024 & $0.360 \pm 0.031$ & \pm 0.005 \\
$\alpha\left(^{\circ}\right)$ & $92.0 \pm 3.4$ & \pm 0.45 & $92 \pm 7$ & \pm 0.85 \\
$\beta\left(^{\circ}\right)$ & $22.0 \pm 0.8$ & \pm 0.17 & $24.7 \pm 1.8$ & \pm 0.4 \\
$\gamma\left({ }^{\circ}\right)$ & $65.6 \pm 3.3$ & \pm 0.38 & $63 \pm 7$ & \pm 0.7 \\
\hline
\end{tabular}

Table 6: Uncertainties of the CKM parameters obtained from the Standard Model and NP fits using the experimental and theoretical information available today (second and fourth columns) and in 2015 (third and fifth columns) as given in ref. [36].

\begin{tabular}{lll}
\hline \hline Parameter & New Physics fit today & New Physics in 2015 \\
\hline$C_{B_{d}}$ & $0.96 \pm 0.23$ & \pm 0.031 \\
$\phi_{B_{d}}\left({ }^{\circ}\right)$ & $-2.9 \pm 1.9$ & \pm 0.4 \\
\hline
\end{tabular}

Table 7: Uncertainties on the New Physics parameters $C_{B_{d}}$ and $\phi_{B_{d}}$ obtained using the experimental and theoretical information available today (left) and in 2015, from ref. [36].
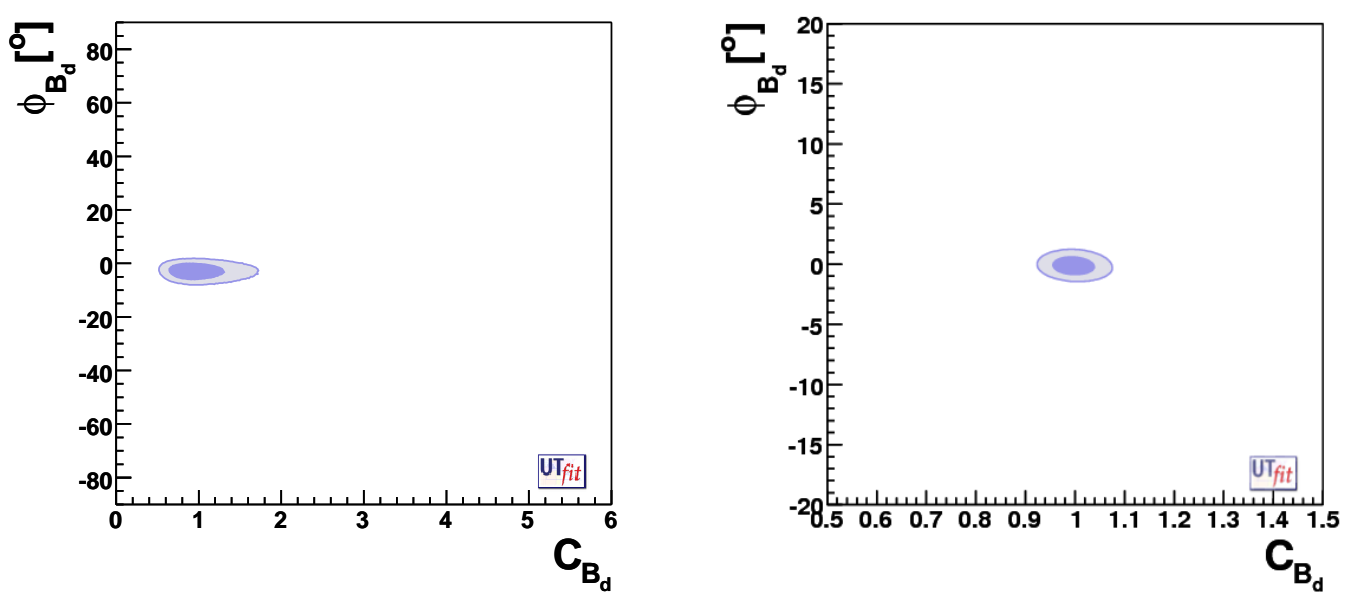

Figure 12: Allowed regions in the $C_{B_{d}}-\phi_{B_{d}}$ plane given by present data (left) and in 2015, from ref. [36]. Note that the scales for the axes are different in the two cases.

be seen, Lattice QCD together with LHCb and SuperB will allow us to probe energy scales much higher than the reach of LHC.

\section{Acknowledgments}

I am indebted to Marcella Bona, Marco Ciuchini, Maurizio Pierini and Viola Sordini for their precious help in preparing this talk. I acknowledge partial support from RTN European contracts 
MRTN-CT-2006-035482 "FLAVIAnet" and MRTN-CT-2006-035505 "Heptools". I am associated to the Dipartimento di Fisica, Università di Roma "La Sapienza".

\section{References}

[1] M. Ciuchini, E. Franco, G. Martinelli, L. Reina and L. Silvestrini, Z. Phys. C 68, 239 (1995) [arXiv:hep-ph/9501265].

[2] D. E. Jaffe and S. Youssef, Comput. Phys. Commun. 101, 206 (1997) [arXiv:hep-ph/9607469].

[3] P. Paganini, F. Parodi, P. Roudeau and A. Stocchi, Phys. Scripta 58, 556 (1998) [arXiv:hep-ph/9711261].

[4] M. Ciuchini et al., JHEP 0107, 013 (2001) [arXiv:hep-ph/0012308].

[5] A. Ali and D. London, arXiv:hep-ph/9405283.

A. Ali and D. London, arXiv:hep-ph/9409399.

A. Ali and D. London, Z. Phys. C 65, 431 (1995) [arXiv:hep-ph/9408332].

A. Ali and D. London, Nuovo Cim. 109A, 957 (1996) [arXiv:hep-ph/9508272].

G. Buchalla, A. J. Buras and M. E. Lautenbacher, Rev. Mod. Phys. 68, 1125 (1996) [arXiv:hep-ph/9512380].

A. J. Buras and R. Fleischer, Adv. Ser. Direct. High Energy Phys. 15, 65 (1998) [arXiv:hep-ph/9704376].

A. Ali and D. London, Eur. Phys. J. C 9, 687 (1999) [arXiv:hep-ph/9903535].

F. Parodi, P. Roudeau and A. Stocchi, Nuovo Cim. A 112, 833 (1999) [arXiv:hep-ex/9903063].

S. Mele, in Proc. of the 5th International Symposium on Radiative Corrections (RADCOR 2000) ed. Howard E. Haber, arXiv:hep-ph/0103040.

M. Bargiotti et al., Riv. Nuovo Cim. 23N3, 1 (2000) [arXiv:hep-ph/0001293].

[6] A. Hocker, H. Lacker, S. Laplace and F. Le Diberder, Eur. Phys. J. C 21, 225 (2001) [arXiv:hep-ph/0104062].

[7] J. Charles et al. [CKMfitter Group], Eur. Phys. J. C 41, 1 (2005) [arXiv:hep-ph/0406184].

[8] M. Bona et al. [UTfit Collaboration], JHEP 0507, 028 (2005) [arXiv:hep-ph/0501199].

[9] A. Abulencia et al. [CDF Collaboration], Phys. Rev. Lett. 97, 242003 (2006) [arXiv:hep-ex/0609040].

[10] E. Gabrielli, A. Masiero and L. Silvestrini, Phys. Lett. B 374, 80 (1996) [arXiv:hep-ph/9509379].

F. Gabbiani, E. Gabrielli, A. Masiero and L. Silvestrini, Nucl. Phys. B 477, 321 (1996) [arXiv:hep-ph/9604387].

[11] J. A. Bagger, K. T. Matchev and R. J. Zhang, Phys. Lett. B 412, 77 (1997) [arXiv:hep-ph/9707225].

[12] M. Ciuchini, E. Franco, V. Lubicz, G. Martinelli, I. Scimemi and L. Silvestrini, Nucl. Phys. B 523, 501 (1998) [arXiv:hep-ph/9711402].

A. J. Buras, M. Misiak and J. Urban, Nucl. Phys. B 586, 397 (2000) [arXiv:hep-ph/0005183].

[13] M. Ciuchini et al., JHEP 9810, 008 (1998) [arXiv:hep-ph/9808328].

[14] D. Becirevic et al., Nucl. Phys. B 634, 105 (2002) [arXiv:hep-ph/0112303]. 
[15] D. Becirevic, V. Gimenez, G. Martinelli, M. Papinutto and J. Reyes, JHEP 0204, 025 (2002) [arXiv:hep-lat/0110091].

S. Aoki et al. [JLQCD Collaboration], Phys. Rev. D 67, 014506 (2003) [arXiv:hep-lat/0208038].

E. Dalgic et al., Phys. Rev. D 76, 011501 (2007) [arXiv:hep-lat/0610104].

[16] A. Donini, V. Gimenez, L. Giusti and G. Martinelli, Phys. Lett. B 470, 233 (1999) [arXiv:hep-lat/9910017].

R. Babich, N. Garron, C. Hoelbling, J. Howard, L. Lellouch and C. Rebbi, Phys. Rev. D 74, 073009 (2006) [arXiv:hep-lat/0605016].

Y. Nakamura et al. [CP-PACS Collaboration], PoS LAT2006, 089 (2006) [arXiv:hep-lat/0610075].

[17] H. Ishino et al. [Belle Collaboration], Phys. Rev. Lett. 98, 211801 (2007) [arXiv:hep-ex/0608035].

A. Kusaka et al. [Belle Collaboration], Phys. Rev. Lett. 98, 221602 (2007) [arXiv:hep-ex/0701015].

A. Somov et al. [Belle Collaboration], Phys. Rev. D 76, 011104 (2007) [arXiv:hep-ex/0702009].

B. Aubert et al. [BABAR Collaboration], Phys. Rev. D 76, 012004 (2007) [arXiv:hep-ex/0703008].

B. Aubert et al. [Babar Collaboration], Phys. Rev. D 76, 052007 (2007) [arXiv:0705.2157 [hep-ex]].

B. Aubert et al. [BABAR Collaboration], arXiv:0807.4226 [hep-ex].

[18] B. Aubert et al. [BABAR Collaboration], Phys. Rev. D 72, 032004 (2005) [arXiv:hep-ex/0504047]. K. Abe et al. [BELLE Collaboration], Phys. Rev. D 73, 051106 (2006) [arXiv:hep-ex/0601032].

A. Poluektov et al. [Belle Collaboration], Phys. Rev. D 73, 112009 (2006) [arXiv:hep-ex/0604054].

B. Aubert et al. [BaBar Collaboration], Phys. Rev. Lett. 99, 251801 (2007) [arXiv:hep-ex/0703037].

B. Aubert et al. [The BABAR Collaboration], Phys. Rev. D 76, 111101 (2007) [arXiv:0708.0182 [hep-ex]].

B. Aubert et al. [BABAR Collaboration], Phys. Rev. D 77, 111102 (2008) [arXiv:0802.4052 [hep-ex]].

K. Abe et al. [Belle Collaboration], arXiv:0803.3375 [hep-ex].

Y. Horii et al. [Belle Collaboration], Phys. Rev. D 78, 071901 (2008) [arXiv:0804.2063 [hep-ex]].

B. Aubert et al. [BABAR Collaboration], Phys. Rev. D 78, 034023 (2008) [arXiv:0804.2089 [hep-ex]].

B. Aubert et al. [BABAR Collaboration], Phys. Rev. D 78, 092002 (2008) [arXiv:0807.2408 [hep-ex]].

[19] B. Aubert et al. [BABAR Collaboration], Phys. Rev. D 71, 112003 (2005) [arXiv:hep-ex/0504035].

B. Aubert et al. [BABAR Collaboration], Phys. Rev. D 73, 111101 (2006) [arXiv:hep-ex/0602049].

F. J. Ronga et al. [BELLE Collaboration], Phys. Rev. D 73, 092003 (2006) [arXiv:hep-ex/0604013]. et al. [Belle Collaboration], arXiv:0809.3203 [hep-ex].

[20] B. Aubert et al. [BABAR Collaboration], Phys. Rev. D 71, 032005 (2005) [arXiv:hep-ex/0411016].

R. Itoh et al. [Belle Collaboration], Phys. Rev. Lett. 95, 091601 (2005) [arXiv:hep-ex/0504030].

[21] B. Aubert et al. [BABAR Collaboration], Phys. Rev. Lett. 99, 081801 (2007) [arXiv:hep-ex/0703019].

[22] K. F. Chen et al. [Belle Collaboration], Phys. Rev. Lett. 98, 031802 (2007) [arXiv:hep-ex/0608039]. et al. [BABAR Collaboration], arXiv:0808.1903 [hep-ex].

[23] M. Ciuchini, M. Pierini and L. Silvestrini, Phys. Rev. Lett. 95, 221804 (2005)

[arXiv:hep-ph/0507290]. 
[24] V. Lubicz and C. Tarantino, arXiv:0807.4605 [hep-lat].

[25] E. Gamiz, arXiv:0811.4146 [hep-lat].

[26] W. Buchmuller and D. Wyler, Nucl. Phys. B 268, 621 (1986).

B. Grinstein and M. B. Wise, Phys. Lett. B 265, 326 (1991).

A. Belyaev and R. Rosenfeld, Mod. Phys. Lett. A 14, 397 (1999) [arXiv:hep-ph/9805253].

R. Barbieri and A. Strumia, Phys. Lett. B 462, 144 (1999) [arXiv:hep-ph/9905281].

R. Barbieri, A. Pomarol, R. Rattazzi and A. Strumia, Nucl. Phys. B 703, 127 (2004) [arXiv:hep-ph/0405040].

[27] C. Csaki, A. Falkowski and A. Weiler, JHEP 0809, 008 (2008) [arXiv:0804.1954 [hep-ph]].

C. Csaki, A. Falkowski and A. Weiler, arXiv:0806.3757 [hep-ph].

A. L. Fitzpatrick, L. Randall and G. Perez, Phys. Rev. Lett. 100, 171604 (2008).

W. F. Chang, J. N. Ng and J. M. S. Wu, arXiv:0809.1390 [hep-ph].

M. Blanke, A. J. Buras, B. Duling, S. Gori and A. Weiler, arXiv:0809.1073 [hep-ph].

K. Agashe, A. Azatov and L. Zhu, arXiv:0810.1016 [hep-ph].

[28] J. M. Soares and L. Wolfenstein, Phys. Rev. D 47, 1021 (1993).

N. G. Deshpande, B. Dutta and S. Oh, Phys. Rev. Lett. 77, 4499 (1996) [arXiv:hep-ph/9608231].

J. P. Silva and L. Wolfenstein, Phys. Rev. D 55, 5331 (1997) [arXiv:hep-ph/9610208].

A. G. Cohen, D. B. Kaplan, F. Lepeintre and A. E. Nelson, Phys. Rev. Lett. 78, 2300 (1997) [arXiv:hep-ph/9610252].

Y. Grossman, Y. Nir and M. P. Worah, Phys. Lett. B 407, 307 (1997) [arXiv:hep-ph/9704287].

[29] M. Bona et al. [UTfit Collaboration], JHEP 0603, 080 (2006) [arXiv:hep-ph/0509219].

[30] M. Bona et al. [UTfit Collaboration], Phys. Rev. Lett. 97, 151803 (2006) [arXiv:hep-ph/0605213].

[31] M. Bona et al. [UTfit Collaboration], JHEP 0803, 049 (2008) [arXiv:0707.0636 [hep-ph]].

[32] M. Bona et al. [UTfit Collaboration], arXiv:0803.0659 [hep-ph].

[33] T. Aaltonen et al. [CDF Collaboration], Phys. Rev. Lett. 100, 161802 (2008) [arXiv:0712.2397 [hep-ex]].

V. M. Abazov et al. [D0 Collaboration], arXiv:0802.2255 [hep-ex].

V. M. Abazov et al. [D0 Collaboration], Phys. Rev. D 74, 092001 (2006) [arXiv:hep-ex/0609014].

V. M. Abazov et al. [D0 Collaboration], Phys. Rev. Lett. 98, 151801 (2007) [arXiv:hep-ex/0701007].

[34] S. Baek, T. Goto, Y. Okada and K. i. Okumura, Phys. Rev. D 63, 051701 (2001)

[arXiv:hep-ph/0002141].

D. Chang, A. Masiero and H. Murayama, Phys. Rev. D 67, 075013 (2003) [arXiv:hep-ph/0205111].

R. Harnik, D. T. Larson, H. Murayama and A. Pierce, Phys. Rev. D 69, 094024 (2004)

[arXiv:hep-ph/0212180].

J. Hisano and Y. Shimizu, Phys. Lett. B 565, 183 (2003) [arXiv:hep-ph/0303071].

[35] M. Ciuchini, E. Franco, A. Masiero and L. Silvestrini, Phys. Rev. D 67, 075016 (2003) [Erratum-ibid. D 68, 079901 (2003)] [arXiv:hep-ph/0212397].

[36] M. Bona et al., arXiv:0709.0451 [hep-ex].

[37] S. Sharpe, talk given at the workshop "Lattice QCD: Present and Future", Orsay (France), April 14-16, 2004. http://events.lal.in2p3.fr/conferences/lqcd/. 